\title{
Longitudinal Assessment of Pain Management Among the Employed Japanese Population with Knee Osteoarthritis
}

This article was published in the following Dove Press journal:

Clinical Interventions in Aging

\author{
Kaname Ueda $\mathbb{D}^{\prime}$ \\ Tomoyuki Takura ${ }^{2}$ \\ Shinji Fujikoshi' \\ Juliana Meyers ${ }^{3}$ \\ Saurabh P Nagar $\mathbb{D D}^{3}$ \\ Hiroyuki Enomoto (iD) \\ 'Medicines Development Unit Japan, Eli \\ Lilly Japan K.K., Kobe, Japan; \\ ${ }^{2}$ Department of Healthcare Economics \\ and Health Policy, University of Tokyo, \\ Tokyo, Japan; ${ }^{3}$ RTI Health Solutions, \\ Research Triangle Park, NC, USA
}

Purpose: To assess comorbidity burden and pain-management patterns among workingaged patients with knee osteoarthritis only $(\mathrm{KOA} / \mathrm{O})$ and patients with knee osteoarthritis plus osteoarthritis at another site $(\mathrm{KOA} /+)$ in Japan.

Patients and Methods: Retrospective claims data analysis was conducted using the Japan Medical Data Center database. Working-aged adults (aged 40 to 71 years) with 5 years of follow-up and diagnosed with knee osteoarthritis (KOA) between January 1, 2011, and December 31, 2012, were evaluated. The first claim with a KOA diagnosis defined the index date. Patients were divided into two mutually exclusive cohorts: KOA/O and KOA/+. Longitudinal pain-management patterns during each year of follow-up were analyzed.

Results: A total of 2542 patients met study criteria: $1575 \mathrm{KOA} / \mathrm{O}$ and $967 \mathrm{KOA} /+$. Mean age and number of comorbidities were higher among the $\mathrm{KOA} /+$ versus $\mathrm{KOA} / \mathrm{O}$ cohort. Pharmaceutical treatment was received by $91.5 \%$ of patients in the KOA/+ compared with $85.1 \%$ of patients in the KOA/O cohort during the first year of follow-up. The most common pharmacological treatment received during the first year of follow-up was either topical or oral nonsteroidal anti-inflammatory drugs for both cohorts. During each year of follow-up, the $\mathrm{KOA} /+$ cohort had greater proportion of patients with at least one health-care encounter (ie, hospital admissions, outpatient and pharmacy visits) and higher direct medical costs compared with the $\mathrm{KOA} / \mathrm{O}$ cohort.

Conclusion: This study demonstrates that a greater proportion of the working population with $\mathrm{KOA} /+$ received pain-related treatment compared with patients with KOA/O. Further studies are necessary to evaluate appropriate pain management for both KOA only and KOA with other sites.

Keywords: knee osteoarthritis, pain management, Japan, working-aged, HCRU

\section{Introduction}

Osteoarthritis (OA) is a widely prevalent chronic and progressive joint disease characterized by cartilage degradation, bone remodeling, osteophyte formation, joint inflammation, and loss of normal joint function, which commonly affects weight-bearing joints such as the knees, hips, and spine as well as hands and feet. $^{1-3}$ OA can affect single or multiple joints, causing acute and chronic pain, swelling, stiffness, restriction of movement, and, in extreme cases, even permanent disability, with corresponding impacts on overall quality of life and functionality. ${ }^{1,2,4}$ Prevalence of radiographically confirmed knee OA (KOA) in Japan has been reported to be $42.0 \%$ in males and $61.5 \%$ in females aged over
Correspondence: Kaname Ueda Medicines Development Unit Japan, Eli Lilly Japan K.K., 5-I-28 Isogamidori, Chuo-ku, Kobe 65I-0086, Japan

Tel +8I-78-242-95I9

Fax +81-78-242-9939

Email ueda_kaname@lilly.com 
40 years. ${ }^{5}$ Previous epidemiological studies have shown that patients aged 50 years and older often experience pain in multiple joints (median of six joints), with pain due to KOA being the most common complaint. ${ }^{6}$ Previous studies have reported that the risk of pain increases dramatically for those patients with KOA with concomitant feet, back, or hip problems compared with patients with KOA only. ${ }^{7}$ A UK-based postal survey of 18,474 adults aged $\geq 50$ years who registered with primary care general practices also reported that as the number of joints affected by OA increases, the likelihood that the disease is debilitating also increases. ${ }^{8}$

At the present time, no definitive treatment option exists for $\mathrm{OA}$ and the severity of the condition differs across individual patients. Current options for OA-related pain management may include pharmacological and nonpharmacological modalities, alone or in combination..$^{9-11}$ Historically, guidelines for the nonsurgical treatment of KOA have stratified patients into groups with single- or multiple-joint OA or with or without the presence of comorbidities; these patient groups highlight the need to assess pain management and comorbidity patterns separately among patients with single-joint and multiple-joint KOA. ${ }^{12,13}$ Core nonpharmacological pain-management modalities may include weight reduction, rehabilitation, and physical therapy, hydrotherapy, aerobic exercises, and yoga. ${ }^{10,11,13}$ Correspondingly, pain relievers, antiinflammatory drugs, corticosteroids, and intra-articular hyaluronic acid (IAHA) injections are some of the most commonly used pharmacological modalities in Japan.

Despite multiple treatment options available for the management of pain associated with OA, treatment satisfaction remains low. A web-based survey evaluated treatment satisfaction in Japanese patients with $\mathrm{KOA}$ and reported a medication treatment satisfaction rate of 50 as measured by Treatment Satisfaction Questionnaire for Medication global score (on a scale of 0 to 100 ; higher scores indicate higher satisfaction). ${ }^{14}$ Early diagnosis and treatment of early-stage $\mathrm{KOA}$ at a younger age become critical to delay the progression of highly degenerative KOA. ${ }^{15}$ While surgical treatment (eg, total knee arthroplasty) may significantly decrease pain symptoms and improve range of motion for patients with $\mathrm{KOA}$, it is recommended only for advanced KOA or when conservative treatment is ineffective considering benefit-risk balances. ${ }^{16,17}$ Early stage KOA, as is commonly seen in the working population, currently has both a lack of evidence and a lack of options for effective pain-management. ${ }^{18}$
Furthermore, with the majority of publications focusing on only single-joint $\mathrm{OA}$, data regarding patients with KOA with multiple-site joint problems are limited. The hypothesis of this study is that patients with multiple-joint KOA will have a greater treatment burden compared with patients with single-joint OA. To address the noted literature gap and to provide data for patients with both singleand multiple-site joint OA, we conducted a real-world retrospective database study in which the primary objectives were to assess the longitudinal use of painmanagement options among working-aged patients with KOA only $(\mathrm{KOA} / \mathrm{O})$ compared with patients with KOA plus $\mathrm{OA}$ at another site $(\mathrm{KOA} /+)$ in Japan. Patient demographic and clinical characteristics as well as longitudinal health care resource utilization and costs were also reported.

\section{Patients and Methods Data Source and Study Design}

Data for this retrospective cohort study were derived from deidentified health insurance claims between January 1, 2010, and December 31, 2017, in the Japan Medical Data Center (JMDC) Claims Database. ${ }^{19}$ The database includes information predominantly from persons of working age (ie, $<65$ years old) employed by middle- to large-sized companies. At the time the study was conducted, the database included $>3$ million unique persons from 2003 onward and represented $\sim 2.5 \%$ of the total population of Japan.

\section{Patient Selection and Study Cohorts}

The cohort was selected from the population of patients aged over 40 years with an "International Classification of Diseases, Tenth Revision, Clinical Modification" (ICD-10CM) diagnosis of KOA (ICD-10-CM code M17) between January 1, 2011, and December 31, 2012. The date of the first observed KOA diagnosis between January 1, 2011, and December 31, 2012, defined the index date. All patients were required to have a minimum of 1 year of enrollment before the index date (ie, baseline period) and 5 years of enrollment after the index date (ie, follow-up period). To ensure sample specificity, patients were further required to have at least one additional KOA diagnosis at any point during the follow-up period and no diagnoses for KOA during the baseline period. To avoid the likelihood of skewed health-care resource use (HCRU) and cost estimates in the two cohorts of interest, patients with 
a diagnosis of cancer during the 5 -year follow-up period were excluded from the study.

This study compared the cohort of patients with KOA/ $\mathrm{O}$ to the cohort of patients with $\mathrm{KOA} /+$. Definitions for these cohorts are as follows:

$\mathrm{KOA} /+$ cohort: patients were included in this cohort if they had at least one diagnosis of OA at another site in the 12 months before or after the index date. ICD-10-CM codes for other OA sites were M16, M18, M19, M40, M41.8, M43.0, M43.1, M47.8, and M47.9.

KOA/O cohort: patients were included in this cohort if they did not have any diagnosis of OA at another site in the baseline and follow-up periods.

As the patient data used in this study were anonymized, the Ethical Guidelines for Medical and Health Research Involving Human Subjects (Ministry of Education, Culture, Sports, Science and Technology and Ministry of Health, Labour and Welfare of Japan) were not applicable to this study.

\section{Study Measures and Definitions}

The study evaluated patient demographics, clinical characteristics, pain-management patterns, HCRU, and direct medical costs. Baseline characteristics measured at the index date included age, sex, and Charlson Comorbidity Index (CCI). ${ }^{20}$ Other comorbidities that may have affected OA-related treatment defined by Osteoarthritis Research Society International (OARSI) guidelines are described in Supplementary Table A-1 and were examined during each year of the follow-up period. ${ }^{13}$ The distribution of physician department (eg, orthopedic surgeons, rheumatologists, anesthesiologists, internal medicine/ general practitioners) and the type of setting (eg, public hospital, university hospital, clinic) for the KOA diagnosis on the index date was reported. Among patients with $\mathrm{OA}$ at other sites within 1 year before and after the index date, the site of other OA diagnosis was reported.

Longitudinal pain-management patterns were assessed for the two cohorts of interest. The number and percentage of patients with broad categories of treatment (ie, nonpharmacological treatment modalities, pharmaceutical treatment, injectable treatment, surgery) at least once during each year of the follow-up period were evaluated. Nonpharmacological treatments included physical therapy and manual/instrumental therapy. Pharmaceutical and injectable (ie, corticosteroids, intra-articular hyaluronic acid, trigger point injection) treatments were identified based on product generic and brand names, as well as therapeutic class descriptions as recorded in the study database. Medical procedures were identified based on unique Japanese procedure codes.
For each patient, all-cause and OA-related HCRU and costs were documented. All-cause HCRU included all claims, regardless of the diagnosis. OA-related hospital admissions were identified by searching for inpatient hospital confinements in which OA was recorded as the primary discharge diagnosis or in which an OA procedure occurred (ie, arthroscopic surgery, osteotomy, arthroplasty, arthrodesis). Osteoarthritis-related outpatient visits were identified by searching for medical claims with any diagnosis (ie, primary or secondary) of OA, an OA procedure, a nonpharmacological treatment modality, or administration of an injectable treatments.

All-cause and OA-related HCRU and costs were estimated during each year of follow-up. HCRU was reported for hospitalizations, outpatient visits, and pharmacy visits and cost components included hospitalizations, medication use, and outpatient visits. Specifically, proportions of patients with an inpatient or outpatient visit were reported. Similarly, the mean number of outpatient visits during the relevant period was reported. Furthermore, medical costs by hospital department (inpatient and outpatient) and medication costs were reported. The costs represent payments for medical services and prescription drugs and included the insurance payment and patient copayment amount. All cost data were reported in Japanese yen $(¥)$ and were inflated to 2017 using the medical care component of the Japanese Consumer Price Index. ${ }^{21}$

\section{Statistical Analysis}

Patient demographics, clinical characteristics, painmanagement patterns, and all-cause and OA-related HCRU and costs were reported for $\mathrm{KOA} / \mathrm{O}$ and $\mathrm{KOA} /+$ cohorts.

Descriptive analyses entailed the tabular display of mean values and standard deviations of continuous variables of interest (eg, HCRU) and frequency distributions for categorical variables (eg, sex). The statistical significance of descriptive differences in patient characteristics, pain-management patterns, and HCRU between KOA/O and KOA/+ cohorts were tested using the Student $t$ test, chi-square test, and Fisher exact test, as appropriate, with results of significance reported. A critical value of 0.05 was used to determine statistical significance. All analyses were conducted using SAS version 9.3 or later (SAS Institute, Inc., Cary, North Carolina).

\section{Results}

A total of 2542 patients met the study criteria: 1575 in the $\mathrm{KOA} / \mathrm{O}$ cohort and 967 in the KOA/+ cohort (Figure 1). Patient demographics are presented in Table 1. Mean age 


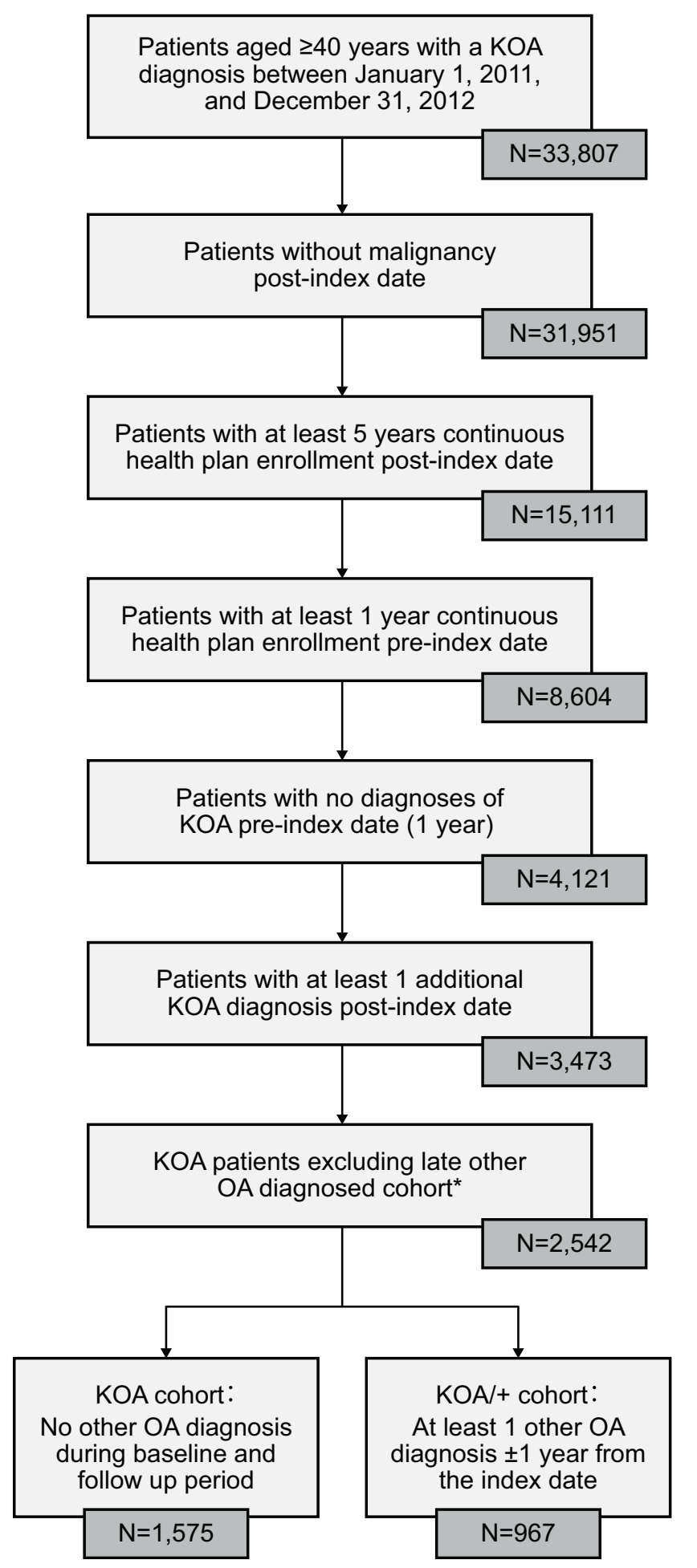

Figure I Sample attrition chart.

Note: *Late other OA diagnosed cohort is defined as no other OA diagnosis during selection window and at least one other $O A$ diagnosis from the second to fifth year from the index date.

Abbreviations: $\mathrm{KOA} / \mathrm{O}$,knee $\mathrm{OA}$ only; $\mathrm{KOA} /+$, knee $\mathrm{OA}$ plus $\mathrm{OA}$ at another site; OA,osteoarthritis.
Table I Patient Demographic and Clinical Characteristics

\begin{tabular}{|c|c|c|}
\hline Characteristics & $\begin{array}{l}\text { KOA/O } \\
(n=1575)\end{array}$ & $K O A /+(n=967)$ \\
\hline Age at index date, mean (SD) & $52.0(6.9)[40: 71]$ & $54.7(7.3)[40: 71]$ \\
\hline [min:max] & & \\
\hline Female, n (\%) & 791 (50.2) & $612(63.3)$ \\
\hline $\mathrm{CCl}$ score, mean (SD) [min:max] & $0.5(1.0)[0.0: 7.0]$ & $0.8(1.1)[0.0: 7.0]$ \\
\hline At least one comorbidity, $\mathrm{n}(\%)$ & $392(24.9)$ & $362(37.4)$ \\
\hline \multicolumn{3}{|c|}{ Physician department for index knee OA diagnosis, $\mathrm{n}(\%)$} \\
\hline Orthopedic surgery & $1206(76.6)$ & $728(75.3)$ \\
\hline Anesthesiology & $7(0.4)$ & $4(0.4)$ \\
\hline $\begin{array}{l}\text { Internal medicine (general } \\
\text { physician) }\end{array}$ & $315(20.0)$ & $205(21.2)$ \\
\hline Other specialties & $47(3.0)$ & $30(3.1)$ \\
\hline \multicolumn{3}{|c|}{ Type of setting for the index KOA diagnosis, $n$ (\%) } \\
\hline Public hospital & $22(1.4)$ & $28(2.9)$ \\
\hline University hospital & $16(1.0)$ & $7(0.7)$ \\
\hline Other hospital & $222(14.1)$ & $149(15.4)$ \\
\hline Clinic & $1315(83.5)$ & $783(81.0)$ \\
\hline \multicolumn{3}{|c|}{ Site of other OA diagnosis, \pm I year of index date, $n$ (\%) } \\
\hline Spine & & $761(78.7)$ \\
\hline Hip & & $120(12.4)$ \\
\hline Ankles & & $63(6.5)$ \\
\hline Unspecified & & $63(6.5)$ \\
\hline Hands & & $40(4.1)$ \\
\hline Elbows & & $32(3.3)$ \\
\hline Fingers & & $19(2.0)$ \\
\hline Shoulders & & $9(0.9)$ \\
\hline Feet & & $4(0.4)$ \\
\hline Other & & $3(0.3)$ \\
\hline $\begin{array}{l}\text { Number of other diagnosed site } \\
\max ]\end{array}$ & mean $(\mathrm{SD})[\mathrm{min}:$ & $1.2(0.4)[1.0: 5.0]$ \\
\hline
\end{tabular}

Notes: Bold values indicate $P<0.05$. Statistical comparisons were made using the Chi-square/Fisher exact test for proportions and Student $t$ test for means. Abbreviations: $\mathrm{CCl}$, Charlson Comorbidity Index; KOA/O, knee OA only; $\mathrm{KOA} /+$, knee OA plus OA at another site; OA, osteoarthritis; SD, standard deviation.

was higher among the $\mathrm{KOA} /+$ (54.7 years) versus the $\mathrm{KOA} / \mathrm{O}$ (52.0 years) cohort, with greater proportion of females in the $\mathrm{KOA} /+$ versus the $\mathrm{KOA} / \mathrm{O}(63.3 \%$ versus $50.2 \%$ ) cohort. Across both cohorts, most patients 

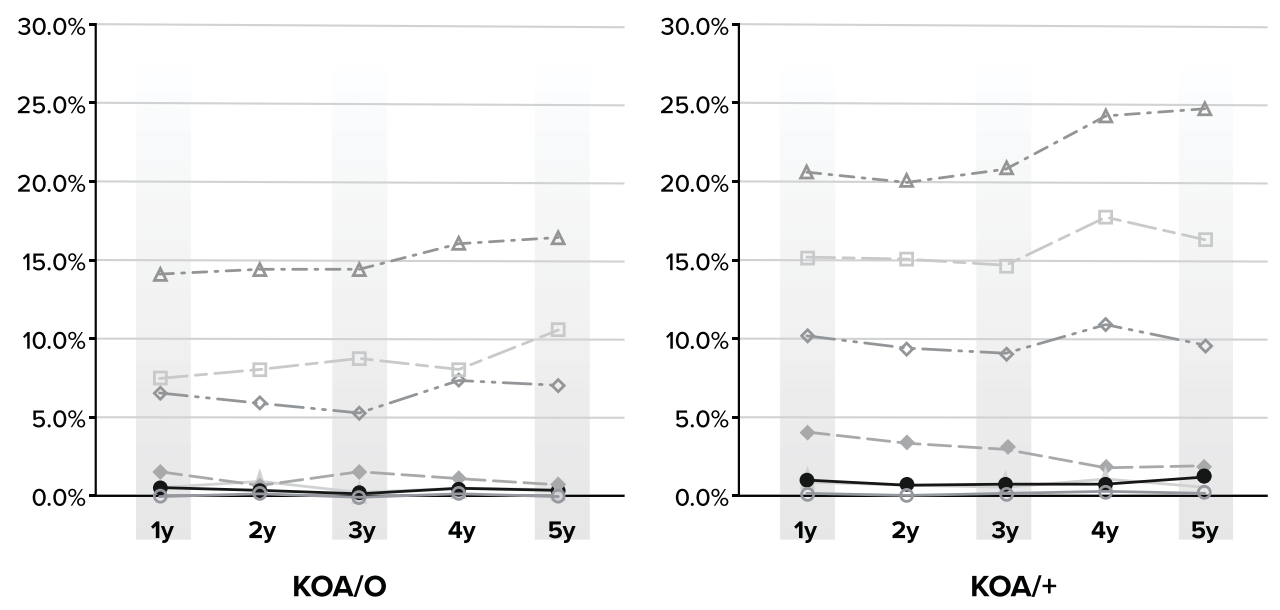

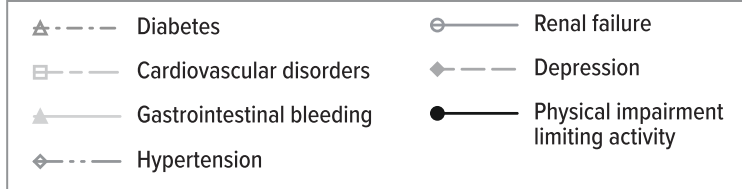

Figure 2 Proportion of Comorbidities to Consider for OA Treatment, Defined by OARSI Guideline.

Abbreviations: $\mathrm{KOA} / \mathrm{O}$, knee $\mathrm{OA}$ only; $\mathrm{KOA} /+$, knee $\mathrm{OA}$ plus $\mathrm{OA}$ at another site; OA, osteoarthritis.

visited orthopedic surgery clinics on the index date. Among patients in the $\mathrm{KOA} /+$ cohort, spine $(78.7 \%)$ and hip $(12.4 \%)$ were the most common sites of other OA.

$\mathrm{KOA} /+$ cohort showed higher mean $\mathrm{CCI}$ score than $\mathrm{KOA} / \mathrm{O}$ cohort (0.8 versus 0.5$)$. A higher proportion of patients had at least one comorbidity during the baseline period that may have been taken into account when deciding on $\mathrm{OA}$ treatment in the $\mathrm{KOA} /+(37.4 \%)$ compared with patients in the KOA/O cohort (24.9\%) (Table 1). The details of reported comorbidities during each year of follow-up period are presented in Figure 2. During the follow-up period, a higher percentage of patients in the $\mathrm{KOA} /+$ than patients with KOA/O cohort had a diagnosis of diabetes, hypertension, cardiovascular disorders, and depression.

Pharmaceutical treatment was received by $91.5 \%$ of patients in the $\mathrm{KOA} /+$ compared with $85.1 \%$ of patients in the KOA/O cohort during the first year of follow-up. The proportion of patients receiving pharmaceutical treatment decreased in the second year of follow-up and remained at approximately the same level for the remainder of the 5-year follow-up period (ie, years 3 through 5). Regardless of the cohort, topical nonsteroidal antiinflammatory drugs (NSAIDs) (received by $82.5 \%$ of patients in the $\mathrm{KOA} /+$ and $74.2 \%$ of patients in the $\mathrm{KOA} / \mathrm{O}$ ), followed by oral non-selective NSAIDs and cyclooxygenase-2 (COX-2) selective inhibitors were the most common pharmaceutical treatments received during the first year of follow-up as well as during the entire follow-up period. Weak opioids were received by $4.3 \%$ of patients in the $\mathrm{KOA} / \mathrm{O}$ and $6.2 \%$ of patients in the $\mathrm{KOA} /+$ cohorts during the first year of the follow-up period, and these percentages did not tend to drastically change over time. Strong opioids were rarely received, regardless of cohort and time period (Table 2).

Injectable treatment was received by more than $40.0 \%$ of patients during the first year of follow-up in both cohorts; IAHA and corticosteroids were the most common injectable treatments received during the first year of follow-up (Table 2). Overall, about $30 \%$ of patients with KOA/O compared with only $15 \%$ of patients with $\mathrm{KOA} /+$ did not receive any treatment between years 2 and year 5 of the follow-up period.

Analysis of the overall treatment regimens and order of therapies received by patients revealed that the most common regimen first received was either topical or oral non-selective NSAIDs (data not shown). A slightly higher mean number of pharmaceutical treatments were received by patients with $\mathrm{KOA} /+$ (3.7) compared with patients with $\mathrm{KOA} / \mathrm{O}$ (3.0) during the entire 5-year follow-up period (data not shown).

All-cause and OA-related resource utilization patterns are presented in Table 3. During each year of the 5-year follow-up period, a higher proportion of patients had at least one OA-related hospitalization among the $\mathrm{KOA} /+$ cohort 
Table 2 Pain-Management Options After Index Date

\begin{tabular}{|c|c|c|c|c|c|c|c|c|c|c|}
\hline \multirow[t]{2}{*}{ Treatment Modality, \% } & \multicolumn{5}{|c|}{ KOA/O $(n=1575)$} & \multicolumn{5}{|c|}{$K O A /+(n=967)$} \\
\hline & I y & $2 y$ & $3 y$ & $4 y$ & 5 y & I y & $2 y$ & $3 y$ & $4 y$ & $5 y$ \\
\hline Nonpharmacological treatment & 26.2 & 18.7 & 16.3 & 16.3 & 16.6 & 44.4 & 39.5 & 35.1 & 32.7 & 38.2 \\
\hline Physical therapy & 12.3 & 7.3 & 7.2 & 6.9 & 7.4 & 24.0 & 20.0 & 16.6 & 16.4 & 18.9 \\
\hline Manual/instrumental therapy & 16.6 & 12.8 & 10.8 & 11.3 & II.I & 27.9 & 25.4 & 24.2 & 21.5 & 25.0 \\
\hline Pharmaceutical treatment & 85.1 & 64.4 & 63.0 & 64.1 & 66.0 & 91.5 & 81.3 & 79.7 & 81.7 & 82.8 \\
\hline Non-selective NSAIDs - oral & 58.8 & 43.5 & 40.8 & 44.3 & 42.4 & 71.8 & 60.3 & 55.2 & 57.6 & 58.2 \\
\hline NSAIDs - topical & 74.2 & 42.4 & 40.1 & 39.6 & 42.8 & 82.5 & 61.9 & 61.2 & 60.3 & 64.9 \\
\hline COX-2 selective inhibitors & 21.8 & 9.2 & 7.7 & 8.0 & 8.8 & 22.9 & 13.9 & 12.4 & 14.6 & 15.0 \\
\hline Acetaminophen & 12.5 & 13.0 & 15.5 & 19.4 & 22.3 & 17.7 & 22.3 & 22.0 & 26.3 & 28.2 \\
\hline Weak opioid & 4.3 & 3.9 & 4.1 & 4.3 & 5.6 & 6.2 & 8.6 & 7.8 & 8.4 & 9.3 \\
\hline Strong opioid & 0.0 & 0.0 & 0.0 & 0.1 & 0.1 & 0.4 & 0.2 & 0.2 & 0.2 & 0.4 \\
\hline Other non-opioid drug & 3.1 & 3.6 & 2.9 & 2.4 & 2.7 & 9.7 & 8.2 & 7.6 & 7.8 & 7.8 \\
\hline SNRI & 1.0 & 0.8 & 1.0 & 0.7 & 1.3 & 1.2 & 1.6 & 1.7 & 1.6 & 2.7 \\
\hline Pregabalin & 1.0 & 1.5 & 1.1 & 1.5 & 1.8 & 6.5 & 6.5 & 6.9 & 6.6 & 8.1 \\
\hline Injectable treatment & 42.0 & 31.7 & 28.6 & 29.0 & 30.8 & 43.5 & 41.6 & 38.5 & 39.5 & 39.0 \\
\hline Corticosteroids & 24.3 & 18.9 & 18.7 & 19.6 & 21.2 & 27.1 & 28.2 & 27.7 & 28.5 & 26.6 \\
\hline IAHA & 33.2 & 19.5 & 16.1 & 15.9 & 17.3 & 31.1 & 23.7 & 21.1 & 21.0 & 21.5 \\
\hline Trigger point injection & 2.3 & 2.2 & 2.4 & 2.3 & 2.8 & 8.0 & 8.1 & 8.0 & 7.8 & 7.7 \\
\hline No pain-related treatment & 12.0 & 30.7 & 32.6 & 31.6 & 30.1 & 7.1 & 15.3 & 16.9 & 15.3 & 14.4 \\
\hline
\end{tabular}

Note: Bold values indicate $P<0.05$.

Abbreviations: COX-2, cyclooxygenase-2; IAHA, intra-articular hyaluronic acid; KOA/O, knee osteoarthritis only; KOA/+, knee osteoarthritis plus osteoarthritis at another site; NSAID, nonsteroidal anti-inflammatory drug; SNRI, serotonin-norepinephrine reuptake inhibitor.

$(3.2 \%, 1.1 \%, 1.7 \%, 2.5 \%, 2.1 \%)$ compared with the KOA/O cohort $(0.9 \%, 0.4 \%, 0.6 \%, 0.4 \%, 0.8 \%)$. A higher number of OA-related outpatient visits were observed among the KOA/ + cohort $(7.5,5.3,5.3,5.4,5.7)$ compared with the KOA/O cohort $(4.7,2.6,2.4,2.4,2.5)$ during each year of the 5-year follow-up period. Similarly, total OA-related direct medical costs were higher among patients in the $\mathrm{KOA} /+$ cohort ( $¥ 206,353$, ¥174,444, ¥181,785, ¥188,829, ¥196,164) compared with the KOA/O cohort ( $¥ 78,350, ¥ 56,924, ¥ 55,816$, $¥ 72,852, ¥ 81,821$ ) during each year of the 5 -year follow-up period. Figure 3 presents data on all-cause and OA-related health-care resource costs.

\section{Discussion}

This study is one of the first to longitudinally assess painmanagement options along with health-care resource use and direct medical costs for the working age population in Japan diagnosed with KOA. A considerable proportion of patients with KOA also had diagnosis of $\mathrm{OA}$ at other sites, and these patients (the KOA/+ cohort) had greater pain-related treatment use and comorbidity burden compared with patients with $\mathrm{KOA} / \mathrm{O}$. In our study, a majority of patients in both cohorts received pharmaceutical treatment during the first year of follow-up. The proportion of patients in our study receiving pharmaceutical treatment decreased between the first year of follow-up and the second year of follow-up and remained stable at this level for the remainder of the follow-up period (ie, years 3 through year 5). Topical and oral NSAIDs were the most frequently observed treatments received for pain management across both the cohorts. Additionally, this study observed that the direct medical cost burden was highest during the first year of follow-up.

Patients in the KOA/+ cohort were observed to have more comorbidities, which may have impacted treatment decisions, during the follow-up periods compared with patients in the KOA/O cohort. Diabetes, hypertension, cardiovascular disorders, and depression were observed in higher proportions of patients with $\mathrm{KOA} /+$ than in those with $\mathrm{KOA} / \mathrm{O}$ 
Table 3 All-Cause and OA-Related Health Care Resource Use

\begin{tabular}{|c|c|c|c|c|c|c|c|c|c|c|}
\hline & \multicolumn{5}{|c|}{ KOA/O (n=I575) } & \multicolumn{5}{|c|}{$K O A /+(n=967)$} \\
\hline & Iy & $2 y$ & $3 y$ & $4 y$ & $5 y$ & Iy & $2 y$ & $3 y$ & $4 y$ & $5 y$ \\
\hline \multicolumn{11}{|l|}{ Patients with services, $\%$} \\
\hline Outpatient visit - OA related & 99.9 & 50.2 & 44.4 & 43.1 & 43.2 & 100.0 & 73.0 & 69.4 & 71.3 & 71.7 \\
\hline Outpatient visit - All cause & 99.9 & 92.7 & 92.0 & 92.4 & 93.1 & 100.0 & 97.8 & 97.5 & 97.9 & 97.8 \\
\hline Pharmacy visit - OA related & 71.8 & 48.6 & 46.3 & 47.1 & 49.1 & 78.5 & 64.8 & 63.8 & 65.5 & 65.1 \\
\hline Pharmacy visit - All cause & 85.2 & 75.6 & 75.4 & 77.7 & 77.8 & 91.6 & 85.6 & 86.0 & 87.8 & 87.8 \\
\hline Hospital admission - OA related & 0.9 & 0.4 & 0.6 & 0.4 & 0.8 & 3.2 & 1.1 & 1.7 & 2.5 & 2.1 \\
\hline Hospital admission - All cause & 2.4 & 2.3 & 2.2 & 2.3 & 3.2 & 4.9 & 2.7 & 4.1 & 4.8 & 4.2 \\
\hline \multicolumn{11}{|l|}{ Number of services, mean (SD) } \\
\hline Outpatient visit - OA related & $4.7(4.2)$ & $2.6(4.1)$ & $2.4(4.2)$ & $2.4(4.2)$ & $2.5(4.4)$ & $7.5(5.7)$ & $5.3(6.0)$ & $5.3(6.2)$ & $5.4(6.0)$ & $5.7(6.3)$ \\
\hline Outpatient visit - All cause & II.I (7.8) & 9.1 (7.9) & $9.2(8.0)$ & $9.3(7.9)$ & $9.7(8.0)$ & $15.8(9.6)$ & $14.2(9.8)$ & |4.| (9.7) & $14.2(9.7)$ & $15.1(10.2)$ \\
\hline Pharmacy visit - OA related & $4.3(0.1)$ & $2.3(0.0)$ & $2.4(0.1)$ & $2.3(0.1)$ & $2.5(0.1)$ & $7.4(10.7)$ & $5.2(0.1)$ & $4.8(0.1)$ & $5.1(0.1)$ & $5.3(0.1)$ \\
\hline Pharmacy visit - All cause & II.3 (8.4) & $8.9(8.2)$ & $9.0(8.5)$ & $9.1(8.3)$ & $9.5(8.5)$ & $15.7(10.3)$ & $14.0(10.8)$ & $13.9(10.6)$ & $14.0(10.5)$ & $14.8(11.0)$ \\
\hline Hospital admission - OA related & $0.0(0.1)$ & $0.0(0.1)$ & $0.0(0.1)$ & $0.0(0.1)$ & $0.0(0.1)$ & $0.1(0.3)$ & $0.0(0.3)$ & $0.0(0.2)$ & $0.0(0.2)$ & $0.0(0.2)$ \\
\hline Hospital admission - All cause & $0.0(0.3)$ & $0.0(0.3)$ & $0.0(0.3)$ & $0.0(0.2)$ & $0.0(0.2)$ & $0.1(0.4)$ & $0.0(0.3)$ & $0.1(0.4)$ & $0.1(0.3)$ & $0.1(0.5)$ \\
\hline
\end{tabular}

Notes: $\mathrm{KOA} / \mathrm{O}, \mathrm{n}=1575 ; \mathrm{KOA} /+, \mathrm{n}=967$.

Abbreviations: $\mathrm{KOA} / \mathrm{O}$, knee $\mathrm{OA}$ only; $\mathrm{KOA} /+$, knee $\mathrm{OA}$ plus $\mathrm{OA}$ at another site; $\mathrm{OA}$, osteoarthritis; SD, standard deviation.

during the follow-up period. A meta-analysis of observational studies reported a higher proportion of comorbidities among patients with KOA compared to those with no KOA. ${ }^{22}$ A recent cross-sectional cohort study conducted in the Netherlands also reported significant comorbid burden among all patients with OA of knee or hip. ${ }^{23}$ Another retrospective database analysis study conducted in the US reported significant comorbidity burden among patients with OA, with approximately $54 \%$ of patients having hypertension and $10 \%$ having cerebrovascular disease. ${ }^{24}$

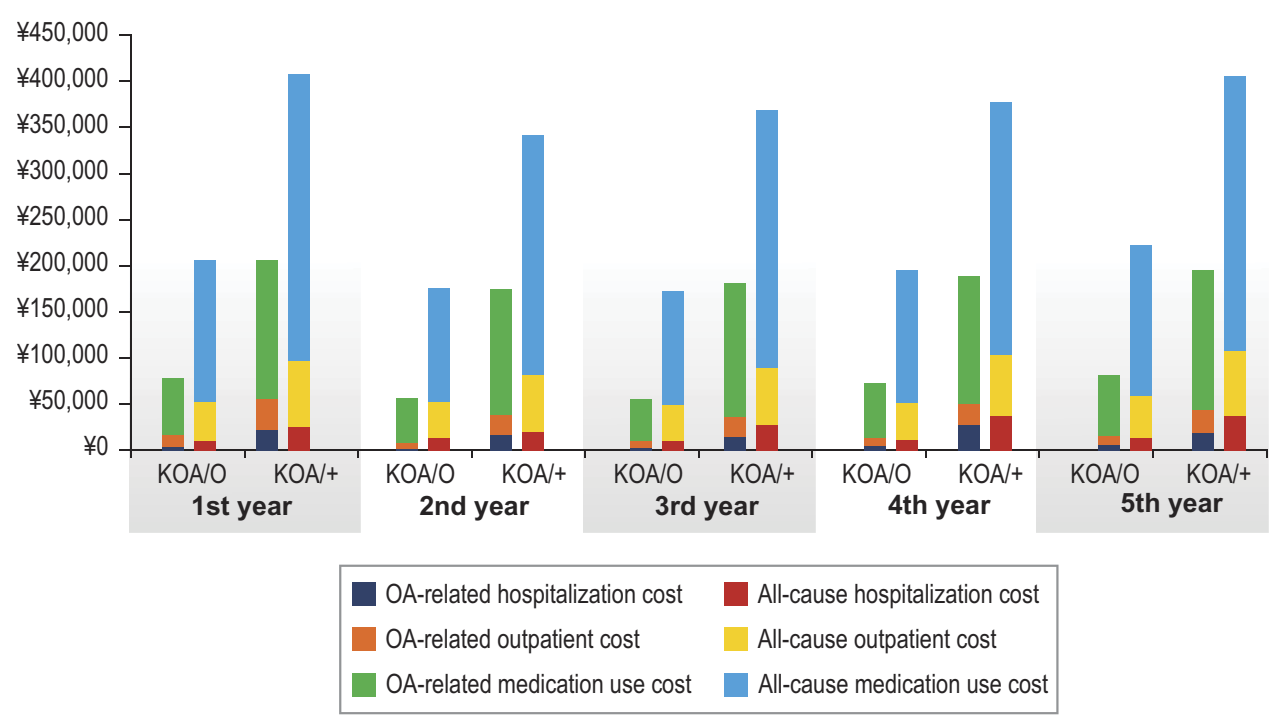

Figure 3 Mean all-cause and OA-related costs estimated during each year of follow-up.

Abbreviations: KOA/O, knee OA only; KOA/+, knee OA plus OA at another site; OA, osteoarthritis. 
To our knowledge, this is the first study reporting the pain-management burden comparing $\mathrm{KOA} / \mathrm{O}$ and $\mathrm{KOA} /+$ among the working age population in Japan. Our research suggests that $55 \%$ of patients with KOA had at least one diagnosis of $\mathrm{OA}$ at another site at any time during the study period. A previous study conducted in a communitybased cohort of patients with radiographically confirmed OA in the US also reported a similar proportion (42.8\%) of patients having multiple-joint disease. ${ }^{25}$ Clinicians should consider focusing on treatments that would benefit multiple-joint $\mathrm{OA}$ along with KOA given the extent of the disease in and the greater health-care burden for the population with multijoint involvement observed in the present analysis. This recommendation is consistent with clinical guidelines that recommend that a multidisciplinary approach should be considered for treatment of OA, specifically $\mathrm{OA}$ involving multiple sites. Multidisciplinary treatments as defined by the National Institute for Health and Care Excellence ${ }^{26}$ are interventions that target at least two different health disciplines (eg, exercise and manual therapy, weight loss, acupuncture, thermotherapy, analgesics, intra-articular injections, surgeries). Arthralgia in Japan is a very common complaint among the elderly population and constitutes a growing segment of Japanese demographics. Furthermore, comorbid chronic diseases and disability have become a major public health concern. ${ }^{27}$ Recent evidence has shown joint disease is the most common reason for entry into certified support level in the long-term care insurance system in Japan. ${ }^{28}$ Further studies are necessary to evaluate appropriate pain management of KOA including OA of other painful sites.

Our study reported that the proportion of patients receiving pharmaceutical treatment decreased between the first year of follow-up and the second year of followup. This observed decrease may be attributed to several factors, one of which may include low pain-related treatment satisfaction among KOA patients in the first year of follow-up. For example, a recent patient survey reported low treatment satisfaction among KOA patients in Japan. ${ }^{14}$ The results of the present analysis may potentially indicate that current treatments may not be providing desired outcomes among KOA patients in Japan, and there is a need for better pain-management options that may in turn improve patient satisfaction.

Overall, higher HCRU and costs were observed among patients with KOA/+ during the follow-up period compared with patients with KOA/O. Wang and colleagues ${ }^{29}$ also reported high medical costs among patients with OA, regardless of site. The present study reported the highest medical costs in the first year after index KOA diagnosis, which could be explained by the more frequent diagnostic and outpatient care visits during the initial year of treatment. Le et $\mathrm{al}^{30}$ conducted a retrospective analysis and reported that patients with newly diagnosed OA incurred higher medical costs compared with patients with existing OA. To our knowledge, few studies have reported medical costs among patients with OA. Le and colleagues ${ }^{30}$ reported mean total all-cause costs of $\$ 19,391$ and OArelated costs of $\$ 6811$ among patients with newly diagnosed OA. Another study conducted in Singapore reported annual direct costs ranging from $\mathrm{S} \$ 1460-\mathrm{S} \$ 7477$ among patients with KOA. ${ }^{31}$ Although the cost burden from other countries cannot be compared with Japan due to variability in health-care systems and differing time periods, in general, OA-related costs reported were high.

There are some limitations to be noted in our study. First, the study sample was selected from enrollees covered by the employees' health insurance system. Because most enrollees are working adults, the proportion of elderly patients aged 65 years and older is low. The current study may be overestimating OA-related costs by incorporating costs attributed to OA-comorbidity-related pharmacy use (eg, serotonin-norepinephrine reuptake inhibitor treatment for depression). Additionally, we required 6 years of continuous health plan enrollment for all patients. Patients in our population may have been healthier than the overall OA population (and correspondingly have had less severe OA than those patients without continuous enrollment for 6 years) as they remained employed for a continuous period of time after their first observed OA diagnosis. Finally, the diagnoses listed in the claims were not validated, and it was not possible to confirm patient diagnoses.

\section{Conclusion}

This study is the first to demonstrate that the working-age population in Japan with $\mathrm{KOA} /+$ received a greater proportion of pain-related treatment compared with patients with KOA/O. Also, this study reports detailed information on patient costs including outpatient visits, inpatient visits, and pharmaceutical treatments filled for patients with $\mathrm{KOA} /+$. The results of the present analysis suggest that multidisciplinary and comprehensive treatment starting at an early stage for not only KOA but also OA at other sites may be important for effective resource utilization in an aging society coping with socioeconomic burdens. 


\section{Disclosure}

Juliana Meyers and Saurabh P. Nagar are full-time employees of RTI Health Solutions, which received funding from Eli Lilly Japan to conduct this research. Kaname Ueda and Shinji Fujikoshi are the full-time employees of Eli Lilly Japan K.K. Hiroyuki Enomoto was a full-time employee of Eli Lilly Japan at the time the study was conducted and is a current employee of Gilead sciences K.K. Tomoyuki Takura received consultant fee from Eli Lilly Japan K.K. The authors report no other conflicts of interest in this work.

\section{References}

1. Thysen S, Luyten FP, Lories RJ. Targets, models and challenges in osteoarthritis research. Dis Model Mech. 2015;8(1):17-30. doi: $10.1242 / \mathrm{dmm} .016881$

2. Xia B, Chen D, Zhang J, Hu S, Jin H, Tong P. Osteoarthritis pathogenesis: a review of molecular mechanisms. Calcif Tissue Int. 2014;95 (6):495-505. doi:10.1007/s00223-014-9917-9

3. Kraus VB, Blanco FJ, Englund M, Karsdal MA, Lohmander LS. Call for standardized definitions of osteoarthritis and risk stratification for clinical trials and clinical use. Osteoarthritis Cartilage. 2015;23 (8):1233-1241. doi:10.1016/j.joca.2015.03.036

4. Yoshimura N, Muraki S, Oka H, et al. Accumulation of metabolic risk factors such as overweight, hypertension, dyslipidaemia, and impaired glucose tolerance raises the risk of occurrence and progression of knee osteoarthritis: a 3-year follow-up of the ROAD study. Osteoarthritis Cartilage. 2012;20(11):1217-1226. doi:10.1016/j.joca.2012.06.006

5. Yoshimura N. Epidemiology of osteoarthritis in Japan: the ROAD study. Clin Calcium. 2011;21(6):821-825.

6. Raja R, Dube B, Hensor EM, Hogg SF, Conaghan PG, Kingsbury SR. The clinical characteristics of older people with chronic multiple-site joint pains and their utilisation of therapeutic interventions: data from a prospective cohort study. BMC Musculoskelet Disord. 2016;17:194. doi:10.1186/s12891-016-1049-0

7. Keenan AM, Tennant A, Fear JO, Emery P, Conaghan PG. Impact of multiple joint problems on daily living tasks in people in the community over age fifty-five. Arthritis Rheum. 2006;55(5):757-764. doi:10.1002/art.22239

8. Thomas E, Peat G, Croft P. Defining and mapping the person with osteoarthritis for population studies and public health. Rheumatology. 2013;53(2):338-345. doi:10.1093/rheumatology/ket346

9. Bhatia D, Bejarano T, Novo M. Current interventions in the management of knee osteoarthritis. J Pharm Bioallied Sci. 2013;5(1):30-38. doi:10.4103/0975-7406.106561

10. Nelson AE, Allen KD, Golightly YM, Goode AP, Jordan JM. A systematic review of recommendations and guidelines for the management of osteoarthritis: the chronic osteoarthritis management initiative of the US bone and joint initiative. Semin Arthritis Rheum. 2014;43(6):701-702.

11. Meneses SR, Goode AP, Nelson AE, et al. Clinical algorithms to aid osteoarthritis guideline dissemination. Osteoarthritis Cartilage. 2016;24(9):1487-1499. doi:10.1016/j.joca.2016.04.004

12. McAlindon TE, Bannuru RR, Sullivan MC, et al. Corrigendum to '2014 OARSI Guidelines for the Non-Surgical Management of Knee Osteoarthritis'[Osteoarthritis and Cartilage 22 (2014) 363-388]. Osteoarthritis Cartilage. 2015;23(6):1026-1034. doi:10.1016/j. joca.2015.02.014

13. Bannuru RR, Osani MC, Vaysbrot EE, et al. OARSI guidelines for the non-surgical management of knee, hip, and polyarticular osteoarthritis. Osteoarthritis Cartilage. 2019;27(11):1578-1589. doi:10.1016/j.joca.2019.06.011
14. Ueda K, Sasaki N, Goren A, et al. Treatment satisfaction with pharmaceutical interventions in Japanese adults with osteoarthritis and chronic knee pain: an analysis of a web-based survey. Clin Interv Aging. 2018;13:2179-2191. doi:10.2147/CIA.S176289

15. Sabatini L, Conti A. Controversies in treatment of early osteoarthritis of the knee. Ann Joint. 2017;2:16. doi:10.21037/aoj.2017.03.03

16. Su X, Li C, Liao W, et al. Comparison of arthroscopic and conservative treatments for knee osteoarthritis: a 5-year retrospective comparative study. Arthroscopy. 2018;34(3):652-659. doi:10.1016/j. arthro.2017.09.023

17. Quinn RH, Murray JN, Pezold R, Sevarino KS. Surgical management of osteoarthritis of the knee. J Am Acad Orthop Surg. 2018;26(9): e191-193. doi:10.5435/JAAOS-D-17-00424

18. Kon E, Filardo G, Drobnic M, et al. Non-surgical management of early knee osteoarthritis. Knee Surg Sports Traumatol Arthrosc. 2012;20(3):436-449. doi:10.1007/s00167-011-1713-8

19. JMDC Claims Database. Japan Medical Data Center website; 2019. Available from: https://www.jmdc.co.jp/en/jmdc-claims-database Accessed April 6, 2019.

20. Charlson ME, Charlson RE, Peterson JC, et al. The Charlson Comorbidity Index is adapted to predict costs of chronic disease in primary care patients. J Clin Epidemiol. 2008;61(12):1234-1240. doi:10.1016/j.jclinepi.2008.01.006

21. Statistics Bureau, Ministry of Internal Affairs and Communications. Consumer price index [Internet]. Statistics Japan. [cited May 1, 2019]. Available from: http://www.stat.go.jp/english/data/cpi/index. htm. Accessed May 18, 2012.

22. Calders P, Van Ginckel A. Presence of comorbidities and prognosis of clinical symptoms in knee and/or hip osteoarthritis: a systematic review and meta-analysis. Semin Arthritis Rheum. 2018;47 (6):805-813. doi:10.1016/j.semarthrit.2017.10.016

23. van Dijk GM, Veenhof C, Schellevis F, et al. Comorbidity, limitations in activities and pain in patients with osteoarthritis of the hip or knee. $B M C$ Musculoskelet Disord. 2008;9(1):95. doi:10.1186/1471-2474-9-95

24. Gore M, Tai KS, Sadosky A, Leslie D, Stacey BR. Clinical comorbidities, treatment patterns, and direct medical costs of patients with osteoarthritis in usual care: a retrospective claims database analysis. J Med Econ. 2011;14(4):497-507. doi:10.3111/13696998.2011.594347

25. Nelson AE, Renner JB, Schwartz TA, Kraus VB, Helmick CG, Jordan JM. Differences in multijoint radiographic osteoarthritis phenotypes among African Americans and Caucasians: the Johnston County Osteoarthritis project. Arthritis Rheum. 2011;63(12):3843-3852. doi:10. 1002/art.30610

26. NICE. Osteoarthritis, Care and Management in Adults. London: NICE; 2014.

27. Aoyagi K, Ross PD, Huang C, Wasnich RD, Hayashi T, Takemoto TI. Prevalence of joint pain is higher among women in rural Japan than urban Japanese-American women in Hawaii. Ann Rheum Dis. 1999;58(5):315-319. doi:10.1136/ard.58.5.315

28. Ministry of Health, Labour and Welfare. Long-term care insurance in Japan. 2019. https://www.mhlw.go.jp/english/topics/elderly/care/2 . html. Accessed April 6, 2019.

29. Wang X, Cicuttini F, Jin X, et al. Knee effusion-synovitis volume measurement and effects of vitamin D supplementation in patients with knee osteoarthritis. Osteoarthritis Cartilage. 2017;25 (8):1304-1312. doi:10.1016/j.joca.2017.02.804

30. Le TK, Montejano LB, Cao Z, Zhao Y, Ang D. Healthcare costs associated with osteoarthritis in US patients. Pain Pract. 2012;12 (8):633-640. doi:10.1111/j.1533-2500.2012.00535.x

31. Xie F, Thumboo J, Fong KY, et al. Direct and indirect costs of osteoarthritis in Singapore: a comparative study among multiethnic Asian patients with osteoarthritis. J Rheumatol. 2007;34(1):165-171. 


\section{Publish your work in this journal}

Clinical Interventions in Aging is an international, peer-reviewed journal focusing on evidence-based reports on the value or lack thereof of treatments intended to prevent or delay the onset of maladaptive correlates of aging in human beings. This journal is indexed on PubMed Central, MedLine, CAS, Scopus and the Elsevier
Bibliographic databases. The manuscript management system is completely online and includes a very quick and fair peer-review system, which is all easy to use. Visit http://www.dovepress.com/ testimonials.php to read real quotes from published authors. 